\title{
Development of KURAMA-II and its operation in Fukushima
}

\section{$\operatorname{AUTHOR}(\mathrm{S})$ :}

Tanigaki, M.; Okumura, R.; Takamiya, K.; Sato, N.; Yoshino, H.; Yoshinaga, H.; Kobayashi, Y.; Uehara, A.; Yamana, $\mathrm{H}$.

\section{CITATION:}

Tanigaki, M. ... [et al]. Development of KURAMA-II and its operation in Fukushima. Nuclear Instruments and Methods in Physics Research Section A: Accelerators, Spectrometers, Detectors and Associated Equipment 2015, 781: 57-64

\section{ISSUE DATE:}

2015-05

URL:

http://hdl.handle.net/2433/194292

\section{RIGHT:}

(C) 2015 Elsevier B.V. NOTICE: this is the author's version of a work that was accepted for publication in Nuclear Instruments and Methods in Physics Research Section A: Accelerators, Spectrometers, Detectors and Associated Equipment. Changes resulting from the publishing process, such as peer review, editing, corrections, structural formatting, and other quality control mechanisms may not be reflected in this document. Changes may have been made to this work since it was submitted for publication. A definitive version was subsequently published in Nuclear Instruments and Methods in Physics Research Section A: Accelerators, Spectrometers, Detectors and Associated Equipment, 781, 57-64l, 2015 doi:10.1016/j.nima.2015.01.086; この論文は出版社版でありません。引用の際には出版社 版をご確認ご利用ください。; This is not the published version. Please cite only the published version. 


\section{Abstract}

7 A carborne survey system, named as KURAMA (Kyoto University RAdia8 tion MApping system), was developed as a response to the nuclear accident 9 at TEPCO Fukushima Daiichi Nuclear Power Plant in 2011. Now the system has evolved into KURAMA-II, characterized by its compactness, autonomous operation, and acquisition of pulse-height spectrum data. A two-year field test of radiation monitoring by KURAMA-II on local buses, performed by Kyoto University, has successfully proceeded to the phase of official operation by the Fukushima prefectural government, supported by Kyoto University and JAEA (Japan Atomic Energy Agency). An outline and the current status of KURAMA-II, including some results of the continuous monitoring by KURAMA-II on local buses in Fukushima, are introduced.

Keywords:

radiometry, mapping, $\gamma$-ray, carborne survey, air dose rate, Fukushima Daiichi nuclear power plant

\section{Introduction}

The magnitude-9 earthquake in eastern Japan and the following massive tsunami caused a serious nuclear disaster of Fukushima Daiichi nuclear power plant. Serious contamination was caused by radioactive isotopes in Fukushima and surrounding prefectures. KURAMA [1] was developed to overcome the difficulties in radiation surveys and to establish air dose-rate maps during and

\title{
Development of KURAMA-II and its Operation in Fukushima
}

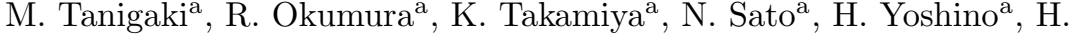 \\ Yoshinaga $^{\mathrm{a}}$, Y. Kobayashi ${ }^{\mathrm{a}}$, A. Uehara ${ }^{\mathrm{a}}, \mathrm{H}$. Yamana ${ }^{\mathrm{a}}$ \\ ${ }^{a}$ Research Reactor Institute, Kyoto University, Kumatori, Osaka 590-0494, Japan
}


after the present incident. KURAMA was designed based on consumer products, and enabled a large number of in-vehicle apparatus to be prepared within a short period owing to its high flexibility in the configuration of data-processing hubs or monitoring cars. KURAMA has been successfully applied to various activities in the radiation measurements and the compilation of radiation maps in Fukushima and surrounding areas.

As the situation was stabilized, the main interest in measurements moves to the tracking of the radioactive materials that have already been released into the environment surrounding the residential areas. KURAMA is not suitable for such purposes. Even though KURAMA enables measurements with a large number of monitoring cars over a wide area at one time, it still requires a trained operator and a driver in each monitoring vehicle. This means that a huge amount of resources and efforts will be required once the surveillance is changed into long-term (several tens years) and detailed monitoring in residential areas. Such monitoring can be realized efficiently if vehicles that periodically move around the residential areas, such as city buses, delivery vans or motorcycles for mail delivery, have compact and full-automated KURAMAs onboard. KURAMA-II is designed for such a purpose.

In this paper, a system outline and the development of KURAMA-II as well as the results of continuous monitoring using KURAMA-II will be introduced.

\section{System outline of KURAMA-II}

The system outline of KURAMA-II is shown in Fig. 1. KURAMA-II basically stands on the architecture of KURAMA [1], but the in-vehicle part has been totally re-designed. In KURAMA, a notebook PC was used in an invehicle unit, but KURAMA-II is based on the CompactRIO series of National Instruments [2] to obtain better toughness, stability and compactness. The 3G/GPS module for CompactRIO by SEA [3] provides time and location data as well as connection to a $3 \mathrm{G}$ network. The radiation detection part has been replaced from the conventional $\mathrm{NaI}$ survey meter to the $\mathrm{C} 12137$ detector series 


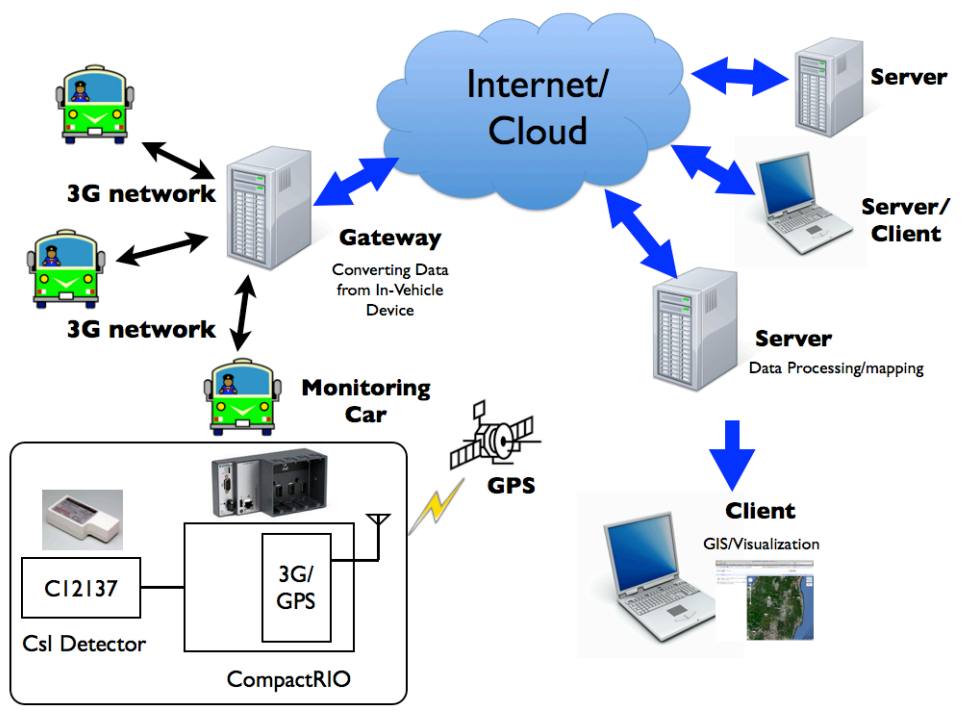

Figure 1: System outline of KURAMA-II. The scheme is basically the same as that of KURAMA, but a gateway server is introduced for the conversion of data chunks from in-vehicle units to data files shared over Dropbox [5] due to no support of Dropbox on CompactRIO.

by Hamamatsu Photonics [4]. This CsI detector series is characterized by its compactness, high efficiency, direct ADC output and USB bus power operation. The direct $\mathrm{ADC}$ output enables one to obtain $\gamma$-ray pulse height spectra during operation. The specifications of C12137 series used in KURAMA-II are summarized in Table 1. All of the components for the in-vehicle part are placed in a small tool box for a better handling (Fig. 2).

Data communication between C12137 and CompactRIO is achieved using the NI-VISA USB RAW mode. C12137 sends a chunk of binary data every 100 ms, consisting of the number of detected $\gamma$-rays, a series of 16-bit ADC data of detected $\gamma$-rays, and the temperature inside C12137. A 4096 ch pulse-height spectrum is constructed every $100 \mathrm{~ms}$ from the obtained 16-bit ADC data inside CompactRIO. $\gamma$-rays whose energy exceeds the upper limit of ADC (around 2.8 $\mathrm{MeV}$ ) are treated as the upper-limit energy of ADC in the construction of pulseheight spectra. 


\begin{tabular}{|c|c|c|}
\hline type & C12137-00 & C12137-01 \\
\hline \hline Scintillator & \multicolumn{2}{|c|}{ CsI(Tl) } \\
\hline Scintillator Size & $13 \times 13 \times 20 \mathrm{~mm}$ & $38 \times 38 \times 25 \mathrm{~mm}$ \\
\hline $\begin{array}{c}\text { Energy Resolution } \\
(662 \mathrm{keV})\end{array}$ & $8 \%$ & $8.5 \%$ \\
\hline $\begin{array}{c}\text { Counting Efficiency }(662 \\
\text { keV }, 0.01 \mu \mathrm{Sv} / \mathrm{h})\end{array}$ & $40 \mathrm{cpm}$ & $400 \mathrm{cpm}$ \\
\hline Detecting Device & \multicolumn{2}{|c|}{$\mathrm{MPPC}$} \\
\hline
\end{tabular}

Table 1: Specification of the C12137 Series [4] used for KURAMA-II. Two types of C12137 series are used, depending on the measurement conditions, such as the expected range of air dose rate.

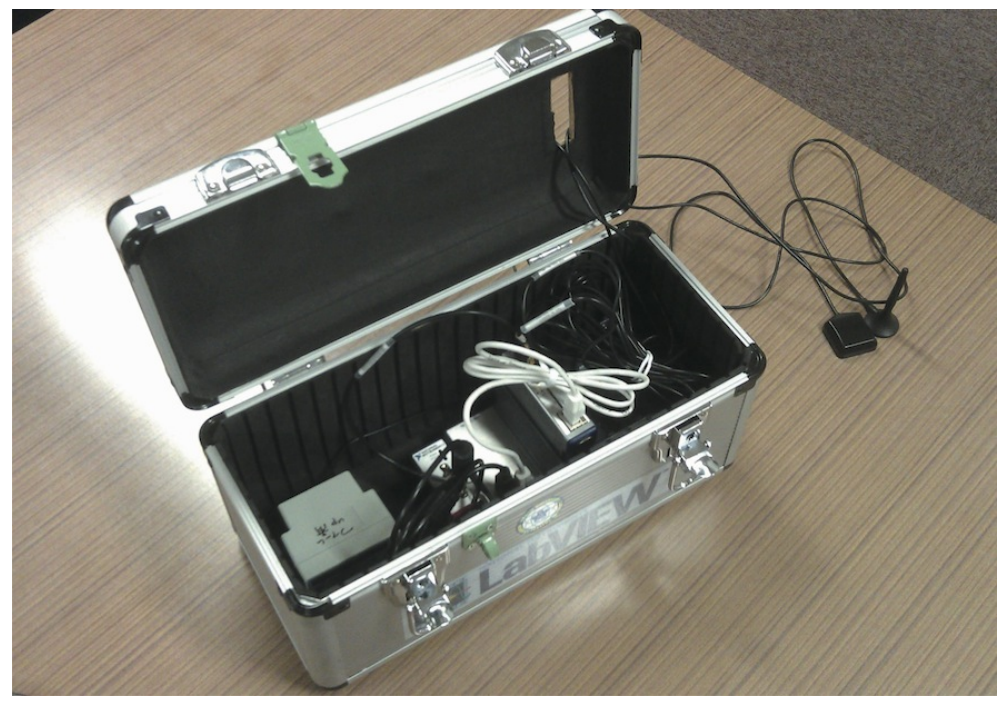

Figure 2: In-vehicle part of KURAMA-II. The components are placed in a small tool box (34.5 $\mathrm{cm} \times 17.5 \mathrm{~cm} \times 19.5 \mathrm{~cm}$ ) for the better handling. This tool box is made of $1 \mathrm{~cm}$ thick balsa wood plates covered with thin aluminum sheets, and no effective shielding towards typical $\gamma$-rays in environment. 
In KURAMA-II, the air dose rate is obtained from the pulse-height spectrum by using spectrum-dose conversion operators, the so-called $G(\mathrm{E})$ function method [6][7]. In this method, the air dose rate is directly obtained from the pulse-height spectrum without any spectrum analysis of the complex spectrum of the environmental $\gamma$-rays. In the $G(\mathrm{E})$ function method, the total dose $\mathrm{D}$ under the existence of a mixed flux of $\gamma$-ray with different energies $E_{0}, E_{1}, E_{2}$, $\ldots\left(\phi\left(\mathrm{E}_{0}\right), \phi\left(\mathrm{E}_{1}\right), \phi\left(\mathrm{E}_{2}\right) \ldots\right)$ is given as

$$
\begin{aligned}
\mathrm{D} & =\sum_{i=0} \phi\left(\mathrm{E}_{i}\right) h\left(\mathrm{E}_{i}\right) \\
& =\sum_{i=0} \int_{0}^{\infty} \phi\left(\mathrm{E}_{i}\right) n\left(\mathrm{E}, \mathrm{E}_{i}\right) G(\mathrm{E}) d \mathrm{E} \\
& =\int_{0}^{\infty} \sum_{i=0} \phi\left(\mathrm{E}_{i}\right) n\left(\mathrm{E}, \mathrm{E}_{i}\right) G(\mathrm{E}) d \mathrm{E} \\
& =\int_{0}^{\infty} N(\mathrm{E}) G(\mathrm{E}) d \mathrm{E},
\end{aligned}
$$

77 under the assumption of the existence of a weighting function, $G(\mathrm{E})$, satisfying 78 the following integral equation:

$$
h\left(\mathrm{E}_{0}\right)=\int_{0}^{\infty} n\left(\mathrm{E}, \mathrm{E}_{0}\right) G(\mathrm{E}) d \mathrm{E}
$$

79 where $h\left(\mathrm{E}_{0}\right)$ is the conversion coefficient for $\gamma$-rays of monochromatic energy so $\mathrm{E}_{0}$, and $n\left(\mathrm{E}, \mathrm{E}_{0}\right)$ is the response function of the detector, $N(\mathrm{E})$ is the measured ${ }_{81}$ pulse-height spectrum, respectively.

82 The Japanese government recommends the ambient dose equivalent, $H^{*}(10)$, 83 as the operational quantity of area monitoring. This time, $G(\mathrm{E})$ functions for ${ }_{84} H^{*}(10)$ were determined by the Japan Atomic Energy Agency (JAEA) group 85 for C12137-00 and C12137-01 (Fig. 3). Details concerning the determination 86 of these $G(\mathrm{E})$ functions and the characteristics of KURAMA-II on radiation 87 detection are available in ref. [8].

${ }_{88}$ KURAMA-II has a built-in function to observe a photo peak in the pulse89 height spectrum, since the gain stability of the detector is directly connected 


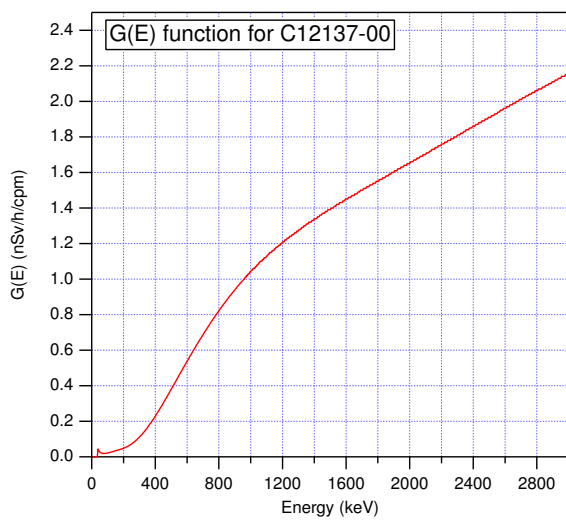

(a) $\mathrm{C} 12137-00$

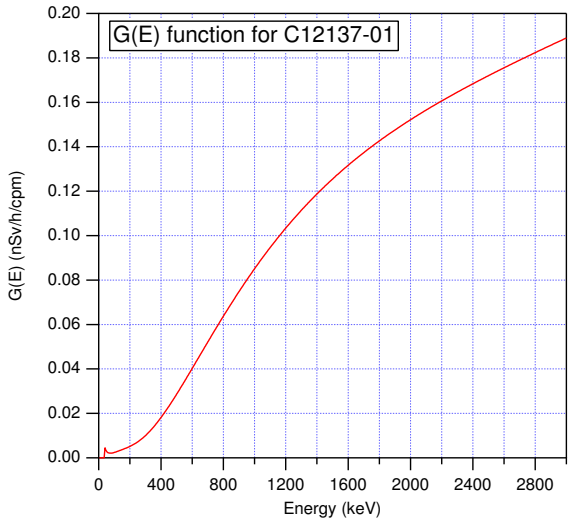

(b) $\mathrm{C} 12137-01$

Figure 3: $G(\mathrm{E})$ functions for (a) C12137-00 and (b) C12137-01 calculated by the JAEA group [8].

to the stability and reliability of the obtained air dose rate. The $796 \mathrm{keV}$ peak of ${ }^{134} \mathrm{Cs}$, which is typically observed as a well isolated peak in the pulse-height spectrum of Fukushima area, is used to monitor the gain shift during operation. Up to now, the peak drift is at most $3 \%$ throughout the operation for one year. This corresponds to $5 \%$ of the drift at most in the air dose rate, one-third of the tolerance for typical portable survey meters used for the air dose rate measurements in Fukushima.

The air dose rate and the pulse-height spectrum for each measurement point are collected in the current KURAMA-II scheme. A simple file-transfer protocol based on RESTful was developed for KURAMA-II, since Dropbox doesn't currently support VxWorks, the operating system of CompactRIO.

In this protocol, a chunk of data as a timestamped file is produced for every three measuring points. Then, every chunk is transferred to a remote "gateway server" by the POST method. The gateway server returns the name list of chunks that are successfully received. The chunks in CompactRIO will not be deleted unless those names are confirmed in the returned list from the gateway server. Unsent chunks are archived at every one hundred of them as a single zip 
file, and these are sent as soon as the network connection is recovered. Timestamped files of the air dose rate and the pulse height spectrum are separately produced. In the case of the air dose rate, Date/Time, location data, the air dose rate, the temperature of the detector, are written into a timestamped text file. Sometimes additional data are also written into this file upon user's request for special purposes. An example of such special data is the air dose rate calculated from the specific energy region, which is requested by one of the user group for their use. The gateway server combines received files to the data file, which is shared by remote servers using a cloud-based file sharing service, Dropbox[5], as was done in original KURAMA. In the case of pulse-height spectrum, the date/time, location data, air dose rate, list of channel numbers and its counts that are non-zero counting are written into a timestamped binary file. The gateway server records the data of the binary files to a SQL database constructed with sqlite3 and spatialite, and the database file is shared over Dropbox. Users can reconstruct the pulse-height spectrum by specifying the time and location on the SQL database (Fig. 4).

\section{Operation of KURAMA-II in Fukushima}

\subsection{Continuous Monitoring by City Buses}

Recent applications of the KURAMA series are mainly with KURAMAII because of its autonomous operation and ease of handling. One of such applications is continuous monitoring with KURAMA-II on city buses (Fig. 5). City buses are suitable for continuous monitoring purpose in residential areas because of their fixed routes in the center of those areas, and their routine operations.

Following the success of a field test in Fukushima city in 2012, the coverage area has been expanded to other major cities in Fukushima, i.e., Koriyama city, Iwaki city, and Aizuwakamatsu city, since the end of December 2012. Four KURAMA-II are assigned to respective cities, and one additional KURAMA-II is assigned to Aizuwakamatsu, mainly for the field tests of prerelease versions of 


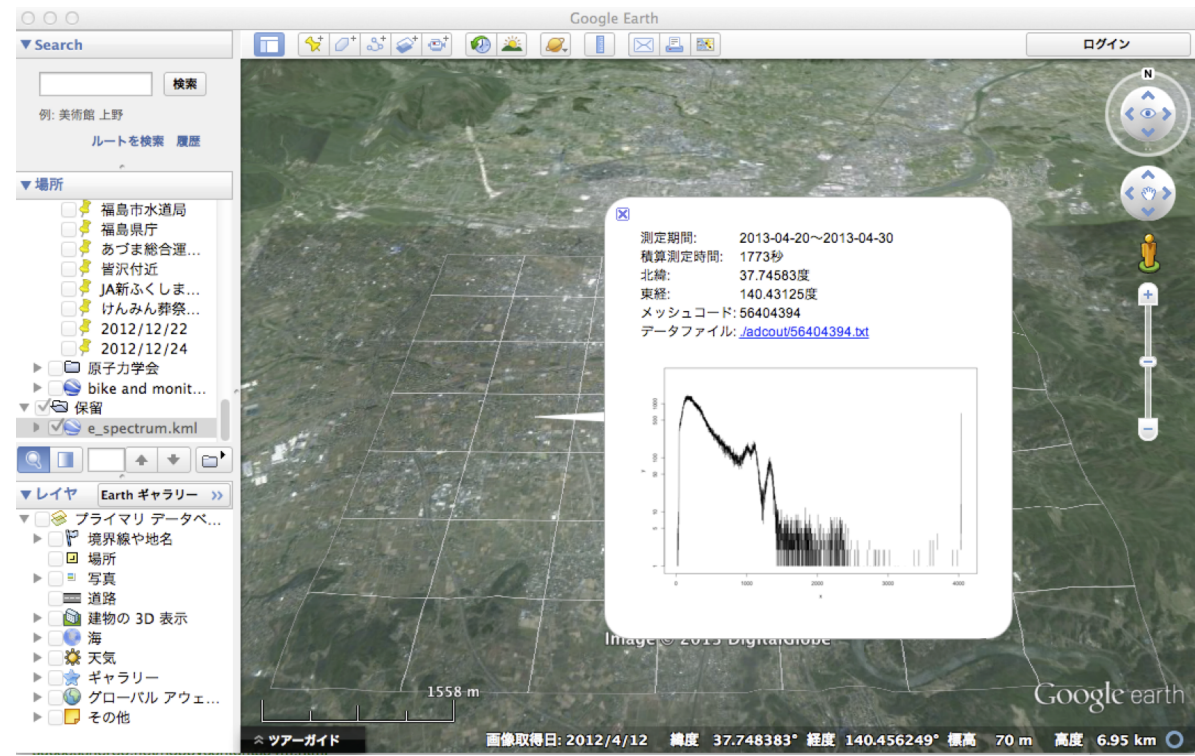

Figure 4: A typical example of the reconstruction of pulse-height spectrum. In this case, the data for a 10 day-period in the spring 2013 and the $1 \mathrm{~km} \times 1 \mathrm{~km}$ grid in Fukushima city is reconstructed. Users can reconstruct a pulse-height spectrum based on their interest by using powerful features on the spatial database of sqlite $3+$ spatialite. 


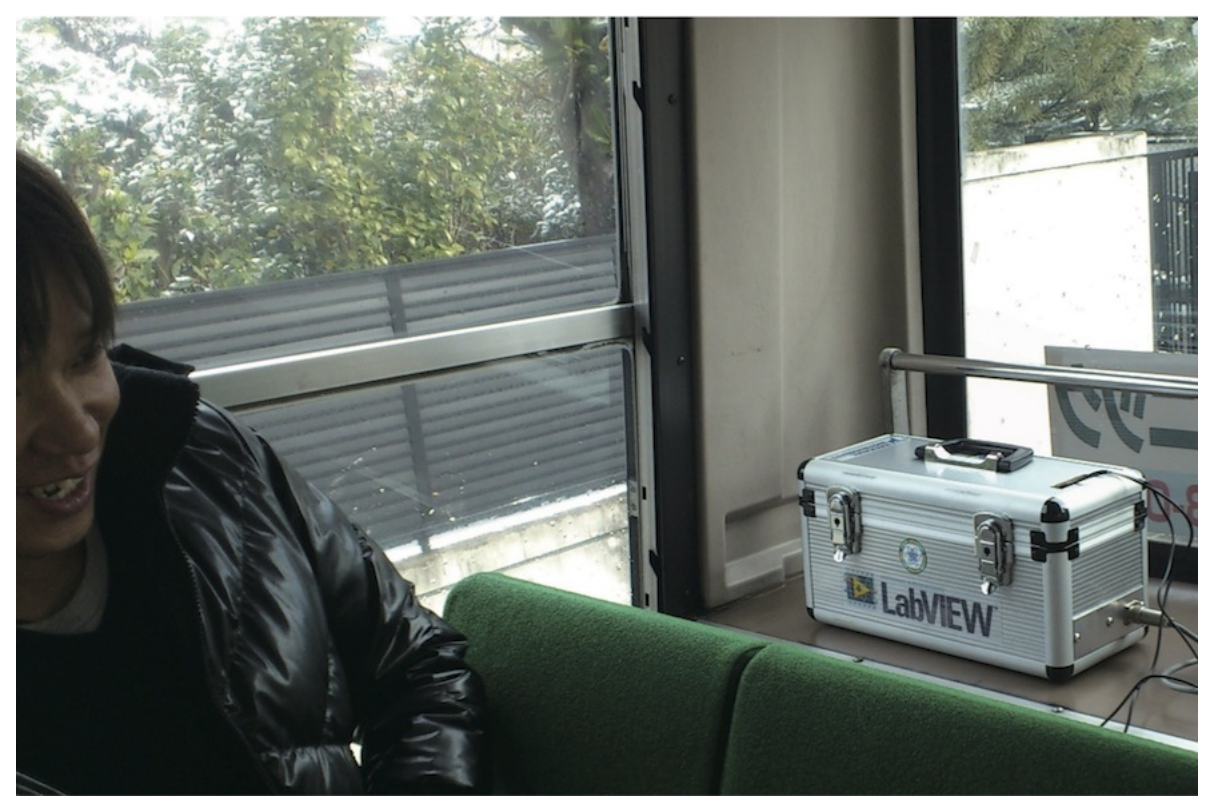

Figure 5: KURAMA-II under a field test on a city bus. An in-vehicle unit is placed on the right side of the rear part in a city bus so that the in-vehicle unit is in the center part of the road. 


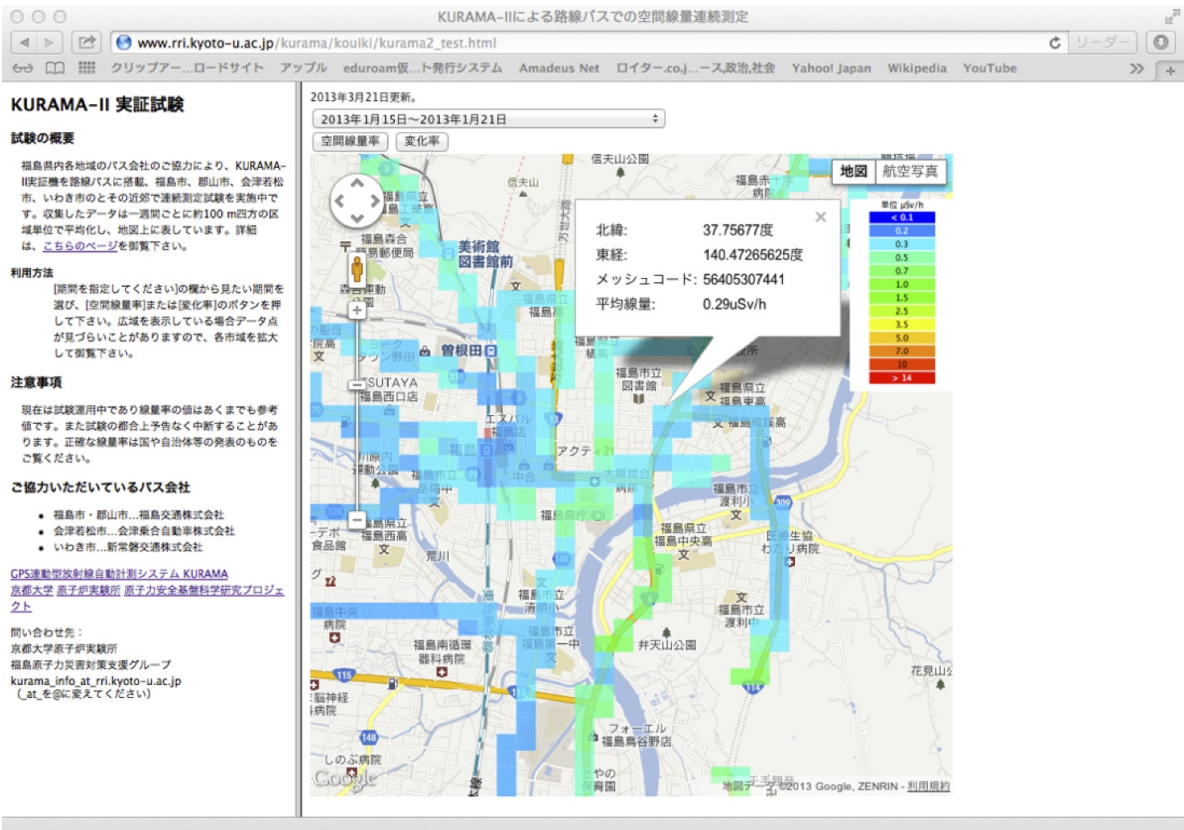

Figure 6: Website for the results of field tests with city buses released from Kyoto University. The results are released to the public on a weekly basis [9].

software. Each KURAMA-II is attached to a certain city bus, and the route of each city bus is determined by the respective bus operator based on their own transportation plans. Typically, each local bus completes most of the possible routes in the respective city within three to five days. The bus routes for respective cities do not overlap each other. The results of these field tests have been released to the public through the web sites (Fig. 6) [9] [10]. The results from this measurement are quite useful for practical purposes, such as confirming the effect of decontamination in residential areas (Fig. 7).

\subsection{Results from the Monitoring by City Buses}

Various analyses are on the way to reveal the trends of the air dose rate, the movement of radioactive materials, and confirmations of the decontamination effects in the coverage area, thanks to the advantages of city buses, i.e., fixed routes and daily operations. For example, the change of the air dose rate from 


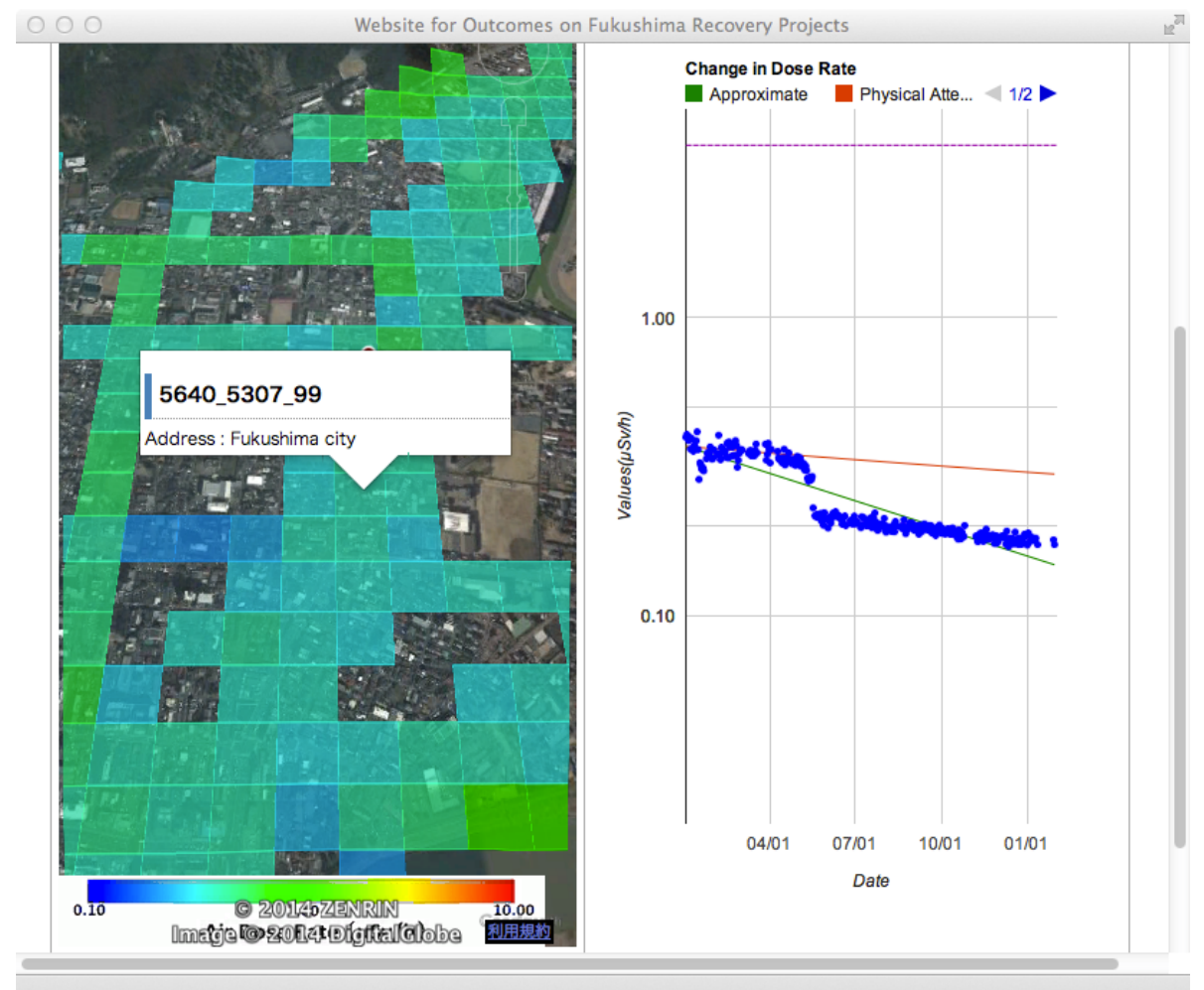

Figure 7: Typical example of the decontamination effect observed through monitoring by KURAMA-II on a local bus. JAEA also releases the monitoring results by KURAMA-II on city buses, including the trends of air dose rates [10]. The grid pointed out in the figure was used as one of the grounds for a large festival in June 2013. The decontamination was performed in May, 2013, prior to the festival. As shown in the graph in this figure, a drastic reduction of air dose rate along with the decontamination was observed, and the decontamination effect has been kept as the reduced air dose rate has been maintained. 
January 2013 to March 2013 was examined for every $100 \mathrm{~m} \times 100 \mathrm{~m}$ grid cell.

In this analysis, the data within every grid cell were averaged for every week, and compared with those in the reference period (from Dec. 20 to 31, 2012). As shown in Fig. 8, the air dose rate is drastically reduced by snow fall. More than a $5 \%$ reduction of air dose rate in average was observed in March, even after the snow effect had disappeared. This implies that the snow water caused the movement of radioactive materials in the environment, such as soils or dead leaves, or the migration of radioactive isotopes into deeper soil layers. There are a few grids in which the air dose rate increased compared to the one in the reference period, indicating the possible concentration of the radioactive materials.

As for the trend in a one-year period, no significant reduction has been observed, except for the effect of snow, but the reduction is found to be generally more rapid than that expected from the physical half lives of Cesium isotopes (Fig. 9). This is also expected to be the result of the movement of radioactive materials or the migration of radioactive isotopes into deeper soil layers; however, further studies should be extended, because the reduction was not increased in the rainy seasons in Fukushima (June, July, September, October) when soils and leaves are expected to be washed away or radioactive materials are expected to migrate into deeper soil layers. One possible hypothesis for this reason is that the feed of radioactive materials from the outside of residential areas (such as mountains) also exists in rainy seasons. Therefore, the systematic tracking of radioactive materials over a wider area should be performed throughout the year.

If we look into the details, the difference in these reductions is found between the areas of higher air dose rate and those of lower air dose rate. In Fig. 10, the reduction in Fukushima city as a typical example of a higher air dose rate (typically $0.2 \sim 0.5 \mu \mathrm{Sv} / \mathrm{h}$ ) and in Aizuwakamatsu city for a lower air dose rate (typically around $0.1 \mu \mathrm{Sv} / \mathrm{h}$ ). Almost no reduction is observed in Aizuwakamatsu, while the reduction in Fukushima city still continues. This trend is reasonably understood as the difference of the responsible radioactive 


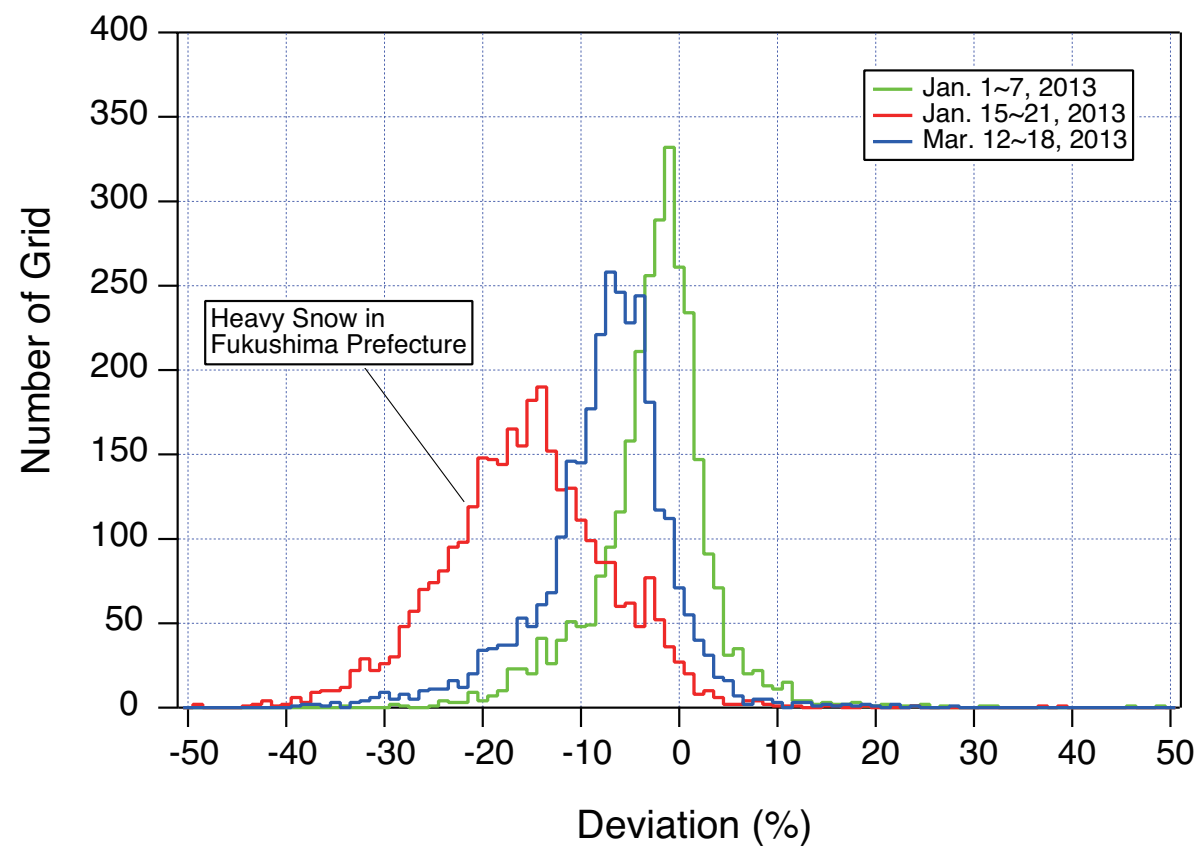

Figure 8: Deviation of the air dose rate in 2013 from the reference period (from Dec. 20 to 31, 2012) in Fukushima monitored by KURAMA-II on city buses. Heavy snow greatly reduced the air dose rate by shielding the radiation.

isotopes for the air dose rate (Fig 11).

\subsection{Periodical Survey by Japanese Government}

The Ministry of Education, Culture, Sports, Science and Technology of Japan (MEXT) has introduced KURAMA-II for the periodic carborne surveys in eastern Japan since March 2012 (Fig. 12) [11]. Around one hundred KURAMA-II were deployed to the local municipalities in eastern Japan in each survey. The staff members in each municipality just attach KURAMA-II into a certain place of conventional sedan cars, and drive around their own municipalities. The results of these periodic surveys are released to the public through the web site [12], and numerical data are released to the researchers as a part of the database of environmental monitoring data [13]. 


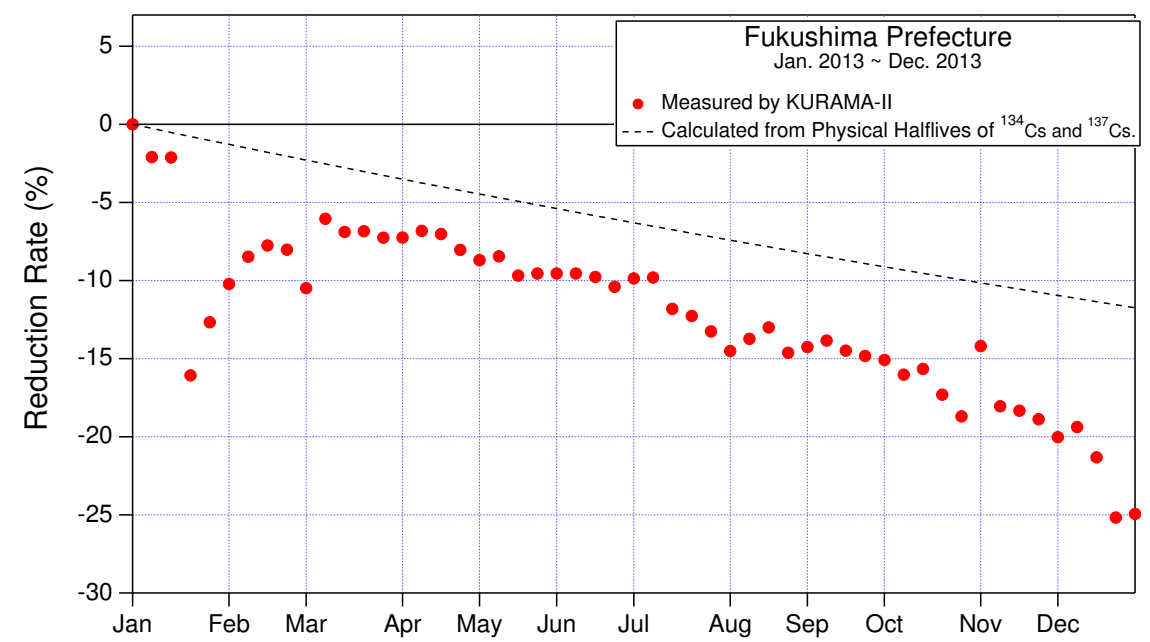

Figure 9: Reduction of the air dose rate in 2013 from the reference period (from Dec. 20 to 31, 2012) in Fukushima monitored by KURAMA-II on city buses. Except for the snow season (from Dec. to Mar.), the reduction is monotonous and more than expected from the physical half-lives of Cesium isotopes.

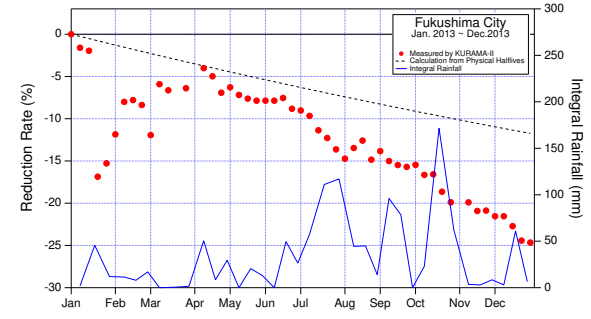

(a) Fukushima city

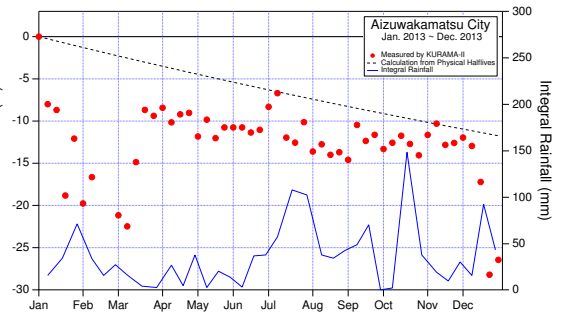

(b) Aizuwakamatsu city

Figure 10: Reduction of the air dose rate from the reference period (from Dec. 20 to 31, 2012) in Fukushima city and Aizuwakamatsu city monitored by KURAMA-II on city buses(red dots) along with the integral rainfall released from the meteorological observatories of respective cities (blue lines). No clear correlation is found between the rain fall and the degree of reduction. Almost no reduction is observed in Aizuwakamatsu city, while that in Fukushima city shows monotonous change, except for the snow season. 


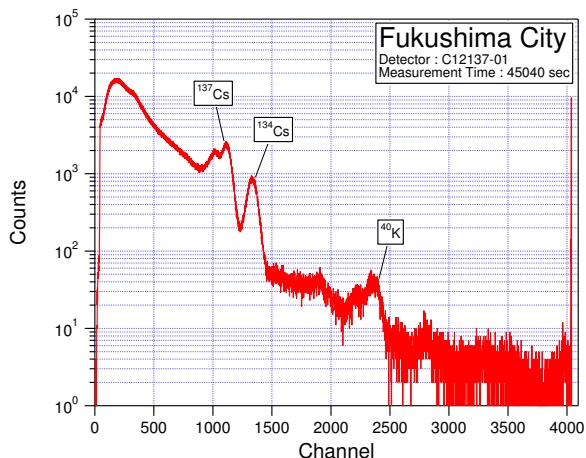

(a) Fukushima city

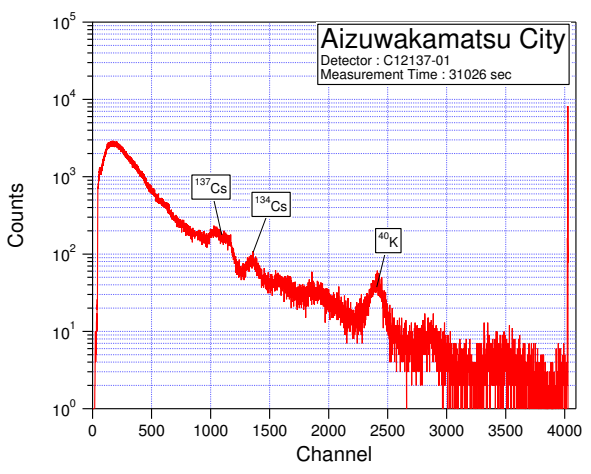

(b) Aizuwakamatsu city

Figure 11: Pulse-height spectra in (a) Fukushima city and (b) Aizuwakamatsu city obtained by KURAMA-II on city buses on 1st Jan. 2014. Typical air dose rates in Fukushima city and Aizuwakamatsu city are $0.2 \sim 0.5 \mu \mathrm{Sv} / \mathrm{h}$ and $0.1 \mu \mathrm{Sv} / \mathrm{h}$, respectively. In Fukushima city, the dominant component of $\gamma$-radiation in environment is Cesium isotopes, while the natural isotopes become competitive to Cesium isotopes in Aizuwakamatsu. The sharp peak at the upper limit of each pulse-height spectrum is due to the folding of pulses more than the upper limit of ADC.

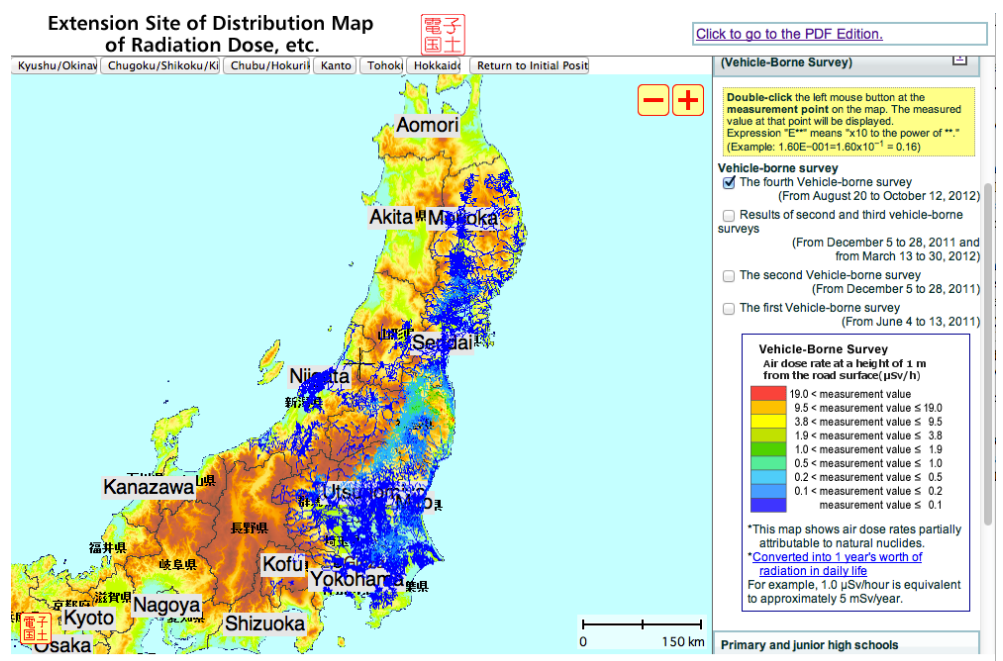

Figure 12: Periodic carborne surveys in the eastern Japan have been performed by MEXT and Nuclear Regulations Authority. The results are available from the interactive web sites [12] [13]. Note that mountain regions are shown in brown in this map. 


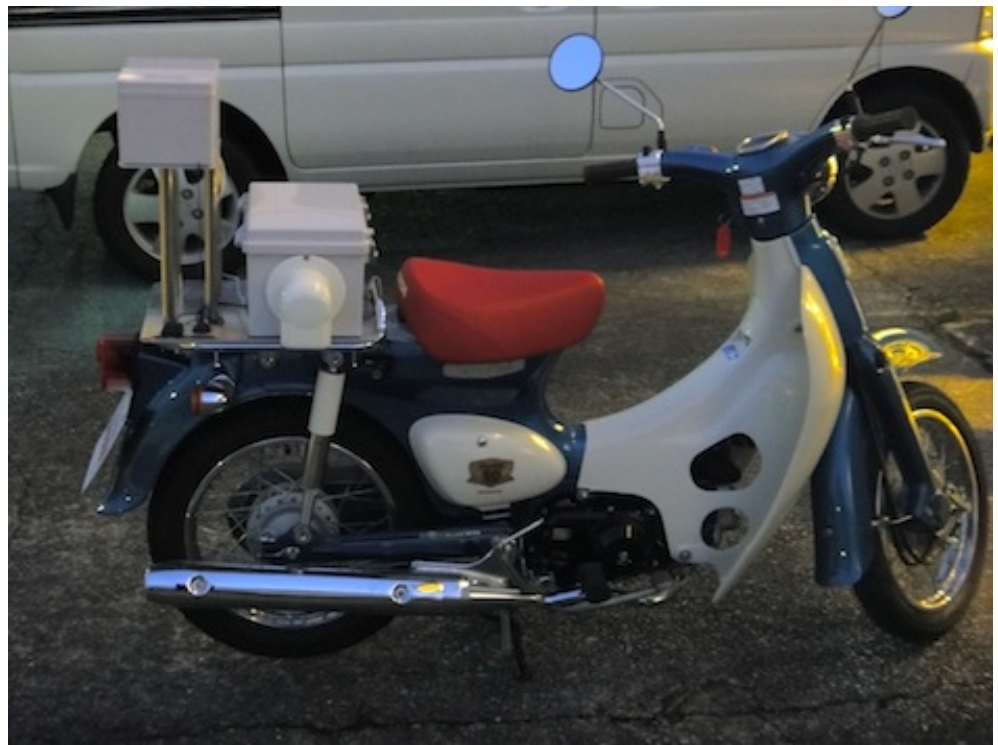

Figure 13: KURAMA-II on a motorcycle. A CsI detector and other components are separately placed in polycarbonate boxes. The height of the detector is adjusted to $1 \mathrm{~m}$ above the ground, so as to meet the standard for the air dose measurement in this accident.

\section{Conclusion and Future Prospects}

KURAMA-II has been developed to enable the long-term monitoring of the air dose rate in residential areas. A test operation throughout the year successfully observed the trends of air dose rates in residential areas, e.g., the shielding effect due to snowfall and reductions due to decontamination activities. Pulseheight spectra obtained by KURAMA-II provide important information concerning radioactive nuclides composing the air dose rates in residential areas. No severe troubles, such as the malfunction of a detector, has been found since the field test in Fukushima was expanded to the major cities in 2012. In-vehicle units work quite stable under the severe vibration and the extreme temperature difference in vehicles. Now troubles are rare and minor ones, such as contact failures of power supply line.

As of July 2014, the monitoring scheme by city buses had just moved into the phase of official operation by the Fukushima prefectural government with 


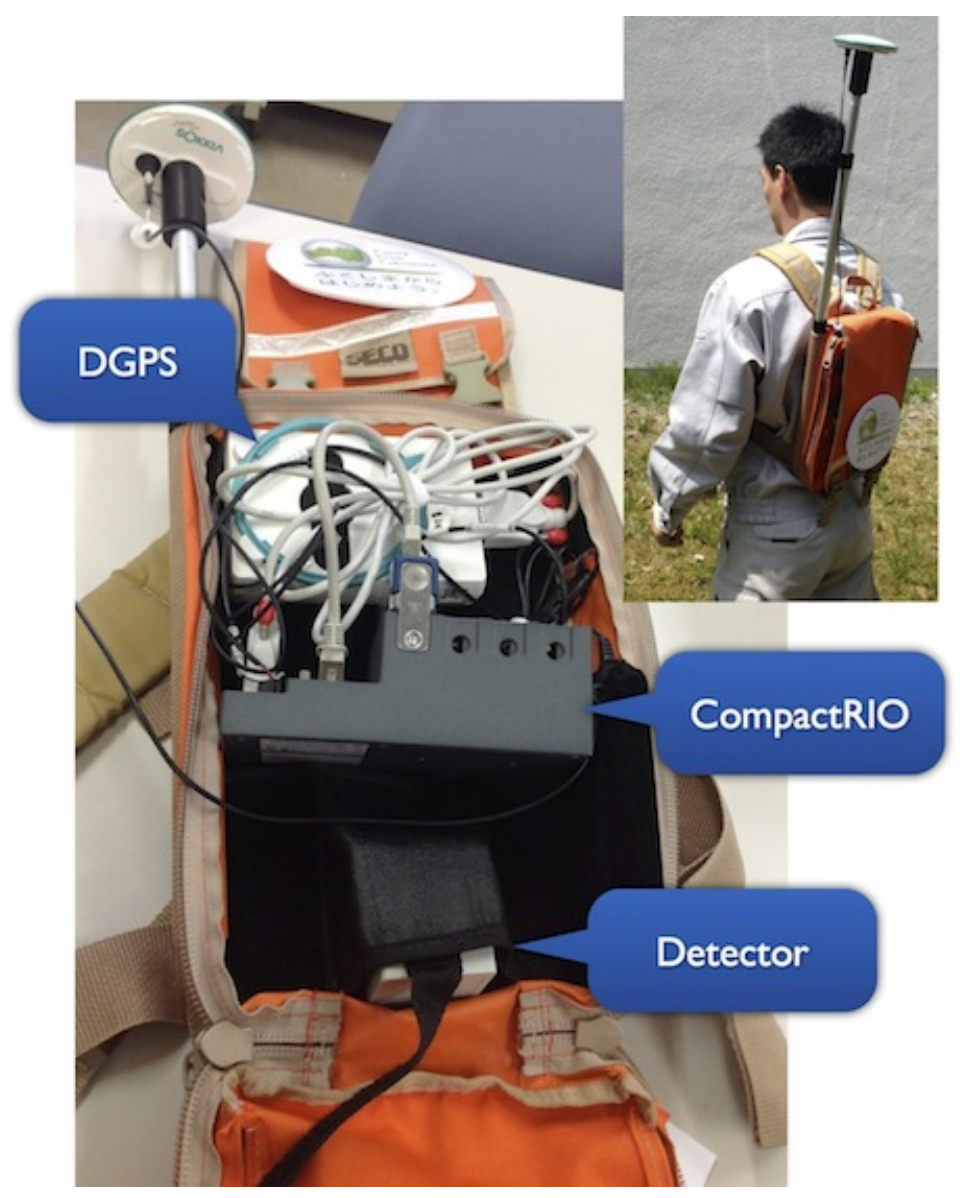

Figure 14: KURAMA-II for the measurement on foot. In this case, a differential GPS (DGPS) unit is used to achieve high accuracy (around $1 \mathrm{~m}$ ) of positioning sufficient for walking survey. 
the collaboration of Kyoto University and JAEA. Thirty city buses owned by local bus companies and twenty official cars owned by Fukushima prefecture are continuously operated throughout Fukushima prefecture. Real time data is released to the public on the display system at the public space of a building in Fukushima city, and the summarized results are available on a weekly basis on the web [14].

Developments for expanding the application of KURAMA-II are presently on the way. For example, the packaging of KURAMA-II is being arranged for surveys by motorcycles or on foot for monitoring, and deployed for measurements in regions where conventional cars can not enter, such as rice fields, forests, and parks (Fig. 13, 14).

\section{Acknowledgements}

The authors are grateful to Dr. Mizuno, Mr. Kimura, Mr. Ito and Mr. Kojima of the Fukushima prefectural government for a continuous support to field tests of KURAMA-II and the establishment of a monitoring scheme based on KURAMA-II on city buses. The authors are indebted to Dr. Saito, Mr. Yoshida and Dr. Takemiya at JAEA for discussions concerning the operation of KURAMA, and to Dr. Tsuda at JAEA for evaluating the $G(\mathrm{E})$ functions of C12137 series. Mr. Nakamura at Hamamatsu Photonics offered the technical support on the C12137 series. Technical support regarding LabVIEW and CompactRIO was served by National Instruments Japan Corporation under a support program named as "Monotsukuri Fukkou Shien Project" by National Instruments Japan Corporation, aiming to help in the recovery from the Great East Japan Earthquake. The authors are grateful to Mr. Matsuura and Mr. Yasuoka at Matsuura Denkosha Corporation for discussions on the KURAMAII source codes. Mr. Muto and Mr. Inomata at Fukushima Transportation Inc., Mr. Suzuki at Shin Joban Kotsu co., ltd., and Mr. Sugihara and Mr. Ishikawa at Aizu Bus Co. Ltd. for their cooperations in the field test of KURAMA-II on city buses. Finally, the authors would like to express their gratitude to Mr. 
and Mrs. Takahashi and the staff members at "Matsushimaya Ryokan", an inn at Iizaka hot spring in Fukushima city, for their heart-warming hospitality and the offer of a foothold for our activities in Fukushima, regardless of the severe circumstances due to the earthquake and the following nuclear accident.

\section{References}

[1] M. Tanigaki, R. Okumura, K. Takamiya, N. Sato, H. Yoshino, H. Yamana, Development of a car-borne survey system, kurama, Nuclear Instruments and Methods in Physics Research Section A: Accelerators, Spectrometers, Detectors and Associated Equipment 726 (2013) 162 - 168.

[2] National Instruments, NI CompactRIO, Web page, 2014. http://www.ni. com/compactrio/.

[3] S. E. A. Datentechnik GmbH, SEA 9724, Web Page, 2014. http://www.sea-gmbh.com/en/products/compactrio-products/ radio-technology/gxxx-3g/.

[4] Hamamatsu Photonics Corporation, Radiation detector modules C12137 series, Catalog, 2013. http://www.hamamatsu.com/resources/pdf/ssd/ c12137_series_kacc1196e01.pdf.

[5] D. Inc., Dropbox, 2014. http://www.dropbox.com.

[6] S. Moriuchi, I. Miyanaga, A SPECTROMETRIC METHOD FOR MEASUREMENT OF LOW-LEVEL GAMMA EXPOSURE DOSE, Health Physics 12 (1966) 541-551.

[7] S. Moriuchi, A Method for Dose Evaluation by Spectrum-Dose Conversion Operator and the Determination of the Operator, JAER 1209, JAERI, 1970.

[8] S. Tsuda, M. Tsutsumi, Calculation and verification of the spectrum-dose conversion operator of various $\mathrm{CsI}(\mathrm{Tl})$ scintillation counters for gamma-ray (in japanese), Jpn. J. Health Phys. 47 (2012) 260 - 265. 
[9] Research Reactor Institute, Kyoto University, Field test of KURAMAII, Web page, 2012. http://www.rri.kyoto-u.ac.jp/kurama/kouiki/ kurama2_test.html.

[10] Sector of Fukushima Research and Development, JAEA, Measurement of air dose rates by route buses in the fukushima prefecture, Web page, 2013. http://info-fukushima.jaea.go.jp/joho_en/project.html.

[11] Ministry of Education and Culture, Sports and Science, Technology, Results of continuous measurement of air dose rates through a vehicle-borne survey (as of march 2012), Press Release, 2012. http://radioactivity. mext.go.jp/en/contents/6000/5637/24/338_Suv_091218_e.pdf.

[12] Ministry of Education and Culture, Sports and Science, Technology, Extension site of the distribution map for radiation dose, 2011. http://ramap. jmc.or.jp/map/eng/.

[13] Nuclear Regulation Authority, Japan, Environment Monitoring Database for the Distribution of Radioactive Substances Released by the TEPCO Fukushima Dai-Ichi NPP Accident, Web page, 2014. https://mapdb. jaea.go.jp/mapdb/en/.

[14] Fukushima Prefectural Government, Vehicle-borne survey monitoring in fukushima prefecture (in japanese), Web page, 2014. https://www.pref . fukushima.lg.jp/sec/16025d/soukou.html. 